Reprod. Nutr. Dévelop., 1983, 23 (4), 717-725.

\title{
Effets d'un régime contenant de l'éthanol sur la composition en acides gras du foie, du sang et du tissu adipeux du rat Zucker fa/fa
}

\author{
Françoise CHANUSSOT, C. KARSENTY, G. DEBRY \\ Département de Nutrition et des Maladies Métaboliques, \\ Université de Nancy l et Groupe de Recherches de Nutrition et Diététique de I'INSERM U. 59, \\ 40, rue Lionnois, 54000 Nancy, France.
}

Summary. Effects of a diet containing alcohol on fatty acid composition of the liver, blood and adipose tissue in Zucker fa/fa rats.

Zucker fa/fa rats were subjected to chronic ethanol intoxication ; (alcohol incorporated into the diet represented $36 \%$ of the energy of the control diet. The amount of endogenous hepatic fatty acids was lower with the alcohol diet than with the control diet.

The incorporated alcohol had no significant influence on the fatty acid composition of adipose tissue. The variations in the fatty acid composition of the blood followed the same patterns as those of the rat liver.

\section{Introduction.}

L'intoxication alcoolique chronique provoque chez le rat normal une stéatose hépatique (Baraona et Lieber, 1979 ; De Carli et Lieber, 1967 ; Lieber, Jones et De Carli, 1965; Reitz, 1979). Toutefois, des travaux antérieurs (résultats non publiés) montrent que, sous l'effet d'une intoxication chronique par l'éthanol, la stéatose hépatique spontanée du rat Zucker fa/fa est moins prononcée qu'avec un régime témoin. L'obésité et l'hyperlipémie du rat obèse sont aussi moins importantes qu'avec le régime témoin. Le but de ce travail est de déterminer comment les modifications qualitatives de la composition en acides gras du foie, du tissu adipeux et du milieu circulant correspondent à une plus faible évolution des syndromes précités du rat $\mathrm{fa} / \mathrm{fa}$ avec le régime alcool, par rapport au régime témoin.

\section{Matériel et méthodes.}

Animaux. - 48 rats mâles adultes (16 rats de souche Wistar, provenant de l'élevage du CESAL - Vigneul sous Montmédy et 32 rats de souche Zucker: $16 \mathrm{fa} / \mathrm{fa}$ obèses et $16 \mathrm{Fa} /$-non obèses provenant de l'élevage du CNRS - 
Orléans La Source) sont placés aléatoirement en cages individuelles à l'animalerie. Ils sont alors soumis à une période d'acclimatation de 2 semaines. Les conditions d'animalerie sont les suivantes : climatisation $=23 \pm 1{ }^{\circ} \mathrm{C}$; lumière artificielle de $7 \mathrm{~h}$ à $19 \mathrm{~h}$; degré hygrométrique contrôlé et maintenu entre 65 et $70 \%$.

Au début de l'expérience, les animaux âgés de 7 à 8 semaines sont répartis en 6 groupes de 8 rats recevant soit un régime témoin $(T)$, soit un régime contenant de l'éthanol (E). La répartition entre les groupes (T) et (E) est effectuée d'une façon aléatoire pour chaque type de rats.

L'évolution du poids des animaux au cours de l'expérience est donnée dans le tableau 1.

Régimes. - Durant la période d'adaptation, les rats sont nourris ad libitum avec un régime standard (UAR $n^{\circ}$ A 04 - Villemoisson sur Orge) dont la composition en protéines, glucides et lipides est respectivement de $17 \mathrm{~g}, 58,7 \mathrm{~g}$ et $3 \mathrm{~g}$ pour $100 \mathrm{~g}$ de régime.

Pendant l'expérience, deux types de régimes isoprotidiques et isolipidiques (en \% énergie) (T et E) sont administrés ad libitum pendant 4 semaines. Ils sont présentés sous forme semi-liquide pour pouvoir incorporer facilement l'éthanol tout en gardant un aspect homogène. Dans le régime $E$, l'éthanol remplace $36 \%$ de l'énergie apportée par l'amidon. Durant les 5 premiers jours, l'éthanol est ajouté en quantités progressivement croissantes aux autres nutriments lors de la préparation quotidienne des régimes : $18 \mathrm{~g} / 100 \mathrm{~g}$ de régime sec les 2 premiers jours, $24 \mathrm{~g} / 100 \mathrm{~g}$ de régime sec les 2 jours suivants, puis $30 \mathrm{~g} / 100 \mathrm{~g}$ de régime sec à partir du $5^{\mathrm{e}}$ jour. La composition des régimes est donnée dans le tableau 2. L'eau de boisson (eau de canalisation) est fournie ad libitum.

Prélèvements. - A l'issue des 4 semaines d'expérience, un rat de chaque lot est sacrifié chaque jour entre 8 h 30 et 10 h 30 , avec permutation circulaire journalière des lots.

Le foie est prélevé très rapidement, pesé et broyé dans un ultraturrax Polytron type PT 10-35, pour réaliser un homogénat dans le tampon saccharose

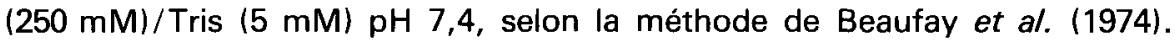

TABLEAU 1

Evolution du poids des animaux (en g)

\begin{tabular}{cccc}
\hline Rats & Régime & $\begin{array}{c}\text { Départ de } \\
\text { l'expérience }\end{array}$ & $\begin{array}{c}\text { Après } 4 \text { semaines } \\
\text { de régime }\end{array}$ \\
\hline \multirow{2}{*}{ Wistar } & $T$ & $254 \pm 7$ & $335 \pm 9$ \\
& E & $260 \pm 5$ & $330 \pm 5$ \\
\hline \multirow{2}{*}{$\mathrm{Fa} /-$} & $T$ & $240 \pm 3$ & $326 \pm 4$ \\
& E & $236 \pm 6$ & $306 \pm 6$ \\
\hline \multirow{2}{*}{$\mathrm{fa} / \mathrm{fa}$} & $T$ & $320 \pm 11$ & $498 \pm 17$ \\
& $\mathrm{E}$ & $314 \pm 9$ & $467 \pm 12$ \\
\hline
\end{tabular}


TABLEAU 2

Composition des régimes.

\begin{tabular}{|c|c|c|c|c|}
\hline & \multicolumn{2}{|c|}{ Régime témoin $(T)$} & \multicolumn{2}{|c|}{ Régime éthanol (E) } \\
\hline & $\underset{\text { régime sec }}{\mathrm{g} / 100 \mathrm{~g}}$ & $\% \mathrm{~kJ}$ & $\underset{\text { régime } \mathrm{g} / 100 \mathrm{~g}}{ }$ & $\% \mathrm{~kJ}$ \\
\hline $\begin{array}{l}\text { Caséine } \\
\text { Lipides (huile d'arachide) } \\
\text { Amidon de maïs } \\
\text { Ethanol } \\
\text { Mélange }{ }^{\left({ }^{a}\right.} \text { ) salin } \\
\text { Mélange }\left({ }^{b}\right) \text { vitaminique } \\
\text { Cellulose }\left({ }^{c}\right) \\
\text { Carraghénates }\left({ }^{d}\right)\end{array}$ & $\begin{array}{r}15,5 \\
19,5 \\
59,5 \\
1,6 \\
1,6 \\
1,3 \\
1\end{array}$ & $\begin{array}{l}13 \\
37 \\
50\end{array}$ & $\begin{array}{r}19,3 \\
24,4 \\
20,7 \\
30 \\
1,5 \\
1,4 \\
1,5 \\
1,2\end{array}$ & $\begin{array}{l}13 \\
37 \\
14 \\
36\end{array}$ \\
\hline Eau (g eau distillée $/ 100 \mathrm{~g}$ régime $\mathrm{sec}$ ) & \multicolumn{2}{|c|}{80} & \multicolumn{2}{|c|}{40} \\
\hline $\mathrm{kJ} / 100 \mathrm{~g}$ de régime sec & \multicolumn{2}{|c|}{1987} & \multicolumn{2}{|c|}{2475} \\
\hline
\end{tabular}

(a) Mélange salin $\left(\mathrm{n}^{\circ}\right.$ 205b UAR - Villemoisson-sur-Orge) $(\mathrm{g} / 100 \mathrm{~g}$ de mélange : $\mathrm{CaHPO}_{4}: 43 \mathrm{~g} ; \mathrm{KCl}: 10 \mathrm{~g} ; \mathrm{NaCl}: 10 \mathrm{~g} ; \mathrm{MgCl}_{2}: 5 \mathrm{~g} ; \mathrm{MgSO}_{4}: 5 \mathrm{~g} ; \mathrm{Fe}_{2} \mathrm{O}_{3}: 0,3 \mathrm{~g} ; \mathrm{FeSO}_{4}, 7 \mathrm{H}_{2} \mathrm{O}:$ $0,5 \mathrm{~g} ; \mathrm{MnSO}_{4}, \mathrm{H}_{2} \mathrm{O}: 0,245 \mathrm{~g} ; \mathrm{CuSO}_{4}, 5 \mathrm{H}_{2} \mathrm{O}: 0,050 \mathrm{~g} ; \mathrm{CoSO}_{4}, 7 \mathrm{H}_{2} \mathrm{O}: 0,410^{-3} \mathrm{~g} ; \mathrm{ZnSO}_{4}, 7 \mathrm{H}_{2} \mathrm{O}:$ $0,2 \mathrm{~g} ; \mathrm{Kl}$ stabilisé : $0,810^{-3} \mathrm{~g} ; \mathrm{NaF}^{4}: 0,025 \mathrm{~g}$.

(b) Mélange vitaminique ( $\mathrm{n}^{\circ} 200$ UAR - Villemoisson-sur-Orge) $(\mathrm{g} / 100 \mathrm{~g}$ de mélange) : Rétinol : $198000 \mathrm{UI}$; Cholécalciférol : $60000 \mathrm{UI}$; Thiamine : $0,2 \mathrm{~g}$; Riboflavine : $0,15 \mathrm{~g}$; Vitamine B3 : $0,35 \mathrm{~g}$; Pyrixodine : $0,1 \mathrm{~g}$; Vitamine $B 7: 1,5 \mathrm{~g}$; Cyanocobalamine $: 0,5 \mathrm{mg}$; Acide ascorbique : 8 $\mathrm{g} ; \alpha$-tocophérol : $1,7 \mathrm{~g}$; Menaphtone $: 0,4 \mathrm{~g}$; Acide nicotinique $: 1 \mathrm{~g}$; Choline : $13,6 \mathrm{~g}$; Acide folique : $0,05 \mathrm{~g}$; Acide paraaminobenzoïque : $0,5 \mathrm{~g}$; Biotine : 0,003 g.

(c) Cellulose Schleicher Schüll-GMBH.

(d) Carrhagénates type I-Sigma.

Le tissu adipeux épididymaire est prélevé sur chaque animal. Le sang est recueilli par ponction de l'aorte abdominale.

Dosages. - Les lipides sont extraits de l'homogénat de foie, du tissu adipeux et du sang total par un mélange de méthylal/méthanol $4 / 1$ selon la méthode de Delsal (1944). Les acides gras sont analysés sous forme d'esters méthyliques, préparés à partir des extraits lipidiques précédents, selon la technique de Morrison et Smith (1964).

L'analyse de ces esters méthyliques d'acides gras est effectué par chromatographie gaz-liquide, le chromatographe étant équipé d'une colonne en acier inoxydable $(2 \mathrm{~mm}$ de diamètre intérieur, longueur $1,20 \mathrm{~m})$ remplie de phase DEGS (a) fixée sur du Chromosorb $W$ lacid washed) 80-100 mesh (b) à un taux de $24 \%$. Les conditions d'analyse sont les suivantes: température de la colonne : $165^{\circ} \mathrm{C}$, de l'injecteur : $230^{\circ} \mathrm{C}$, du détecteur : $210^{\circ} \mathrm{C}$. Le débit d'azote à travers la colonne est de $26 \mathrm{ml} / \mathrm{min}$. La précision des mesures est de $4 \%$.

Les acides gras du foie, du tissu adipeux épididymaire et du sang sont analysés individuellement pour chacun des rats. La comparaison des résultats est effectuée par le test $\mathrm{t}$ de Student.

(a) Applied Science Laboratories, State College, Pensylvania.

(b) Johns Manville, New York, N.Y.

Reproduction Nutrition Développement, $n^{\circ} 4-1983 .-6$. 


\section{Résultats.}

Les résultats sont donnés dans les tableaux 2 et 3 et sur les figures 1, 2 et 3 (\% molaire des acides gras).

Acides gras constitutifs du foie (fig. 1). - La composition en acides gras des lipides hépatiques varie en fonction de la nature du régime et du génotype. Avec le régime témoin, les pourcentages des acides palmitique et oléique sont significativement plus élevés chez le rat $\mathrm{fa} / \mathrm{fa}$ que chez les rats témoins et les taux des acides gras polyinsaturés sont diminués significativement. Les valeurs des rapports $(16: 1)+(18: 1) /(16: 0)+(18: 0)$ et $(20: 4) /(18: 2)$ sont plus élevés dans le foie du rat obèse que dans celui des rats témoins (sauf pour le rapport $(20: 4) /(18: 2)$ du rat $\mathrm{Fa} /-)$.

Le régime alcool entraîne dans le foie du rat obèse, par rapport au régime témoin :

- la diminution du taux des acides palmitique et oléique (variation significative) et de la valeur du rapport $(16: 1)+(18: 1) /(16: 0)+(18: 0)$ (tabl. 3) ;

- l'augmentation du taux des acides gras polyinsaturés (acides linoléique et arachidonique) et de la valeur du rapport (20:4) / (18:2) (tabl. 4).

Les quantités totales des acides gras hépatiques, calculées à partir des quantités de lipides hépatiques (résultats non publiés) sont toujours plus faibles avec le régime alcool qu'avec le régime témoin (tabl. 5).

Acides gras constitutifs du sang (fig. 2). - Comme dans le foie, la composition en acides gras des lipides sanguins varie en fonction de la nature du régime et du génotype.

\section{TABLEAU 3}

Valeurs des rapports des pourcentages molaires $(16: 1)+(18: 1) /(16: 0)+(18: 0)$ dans le foie, le sang et le tissu adipeux épididymaire.

\begin{tabular}{lccccccccc}
\hline & \multicolumn{3}{c}{ Foie } & \multicolumn{3}{c}{ Sang } & \multicolumn{3}{c}{ Tissu adipeux épididymaire } \\
\cline { 2 - 11 } \multicolumn{1}{c}{ Rat } & Wistar & $\mathrm{Fa} /-$ & $\mathrm{fa} / \mathrm{fa}$ & Wistar & $\mathrm{Fa} /-$ & $\mathrm{fa} / \mathrm{fa}$ & Wistar & $\mathrm{Fa} /-$ & $\mathrm{fa} / \mathrm{fa}$ \\
\hline Régime T & 0,70 & 0,53 & 0,86 & 1,09 & 0,59 & 0,84 & 2,12 & 1,98 & 1,63 \\
Régime E & 0,79 & 0,44 & 0,67 & 0,69 & 0,37 & 0,75 & 2,15 & 1,93 & 1,60 \\
\hline
\end{tabular}

\section{TABLEAU 4}

Valeurs des rapports des pourcentages molaires (20:4) / (18:2) dans le foie et le sang.

\begin{tabular}{|c|c|c|c|c|c|c|}
\hline \multirow[b]{2}{*}{ Rat } & \multicolumn{3}{|c|}{ Foie } & \multicolumn{3}{|c|}{ Sang } \\
\hline & Wistar & $\mathrm{Fa} /-$ & $\mathrm{fa} / \mathrm{fa}$ & Wistar & $\mathrm{Fa} /-$ & $\mathrm{fa} / \mathrm{fa}$ \\
\hline $\begin{array}{l}\text { Régime T } \\
\text { Régime E }\end{array}$ & $\begin{array}{l}0,74 \\
0,51\end{array}$ & $\begin{array}{l}1,26 \\
1,25\end{array}$ & $\begin{array}{l}0,89 \\
0,95\end{array}$ & $\begin{array}{l}0,57 \\
0,87\end{array}$ & $\begin{array}{l}0,92 \\
1,58\end{array}$ & $\begin{array}{l}1,03 \\
0,58\end{array}$ \\
\hline
\end{tabular}


TABLEAU 5

\begin{tabular}{lcccccc}
\hline $\begin{array}{c}\text { ( } \mu \text { moles) } \\
\text { Foie entier }\end{array}$ & $\begin{array}{c}\text { Acides } \\
\text { gras totaux }\end{array}$ & $\begin{array}{c}\text { Acide } \\
\text { palmitique }\end{array}$ & $\begin{array}{c}\text { Acide } \\
\text { stéarique }\end{array}$ & $\begin{array}{c}\text { Acide } \\
\text { oléique }\end{array}$ & $\begin{array}{c}\text { Acide } \\
\text { linoléique }\end{array}$ & $\begin{array}{c}\text { Acide } \\
\text { arachidonique }\end{array}$ \\
\hline Régime T & 7192 & 2316 & 719 & 2590 & 755 & 683 \\
Régime E & 4236 & 1123 & 678 & 1237 & 551 & 517 \\
\hline
\end{tabular}

Avec le régime témoin, les pourcentages des acides palmitique et oléique du rat obèse sont plus élevés ou comparables à ceux des rats témoins. Les pourcentages des acides linoléique et arachidonique du rat $\mathrm{fa} / \mathrm{fa}$ sont plus faibles que ceux des rats Wistar et $\mathrm{Fa} /$ - . Toutefois, la valeur du rapport $(20: 4) /(18: 2)$ est plus grande chez le rat obèse que chez les deux autres phénotypes des rats.
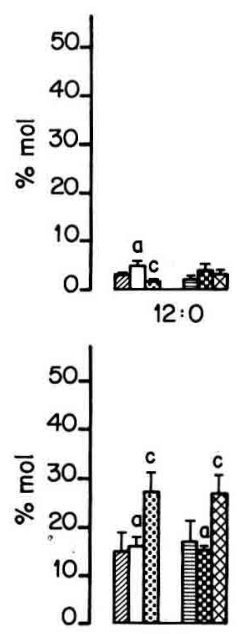

16:0

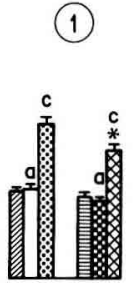

$16: 0$

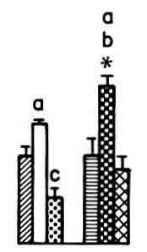

18:0

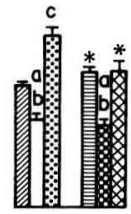

$18: 1$

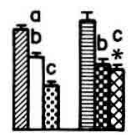

18: 2

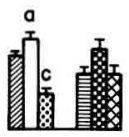

20:4

(2)

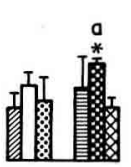

18:0

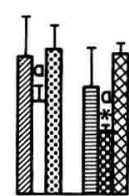

$18: 1$

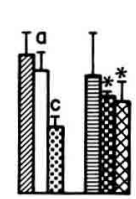

$18: 2$

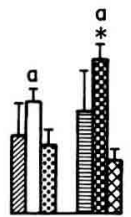

$20: 4$

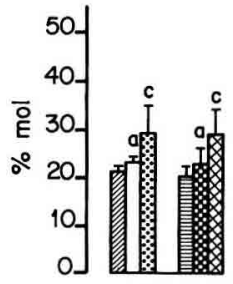

16:0
(3)

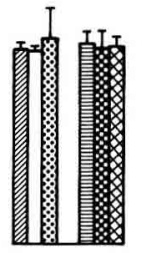

18:1

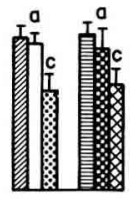

$18: 2$

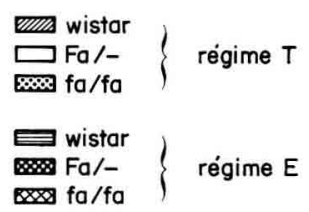

$\left.\begin{array}{l}\mathrm{Fa} /- \\ \mathrm{fa} / \mathrm{fa}\end{array}\right\}$ régime $\mathrm{E}$

Les résultats sont exprimés sous forme de moyennes $\pm \operatorname{ESM}(\mathbf{n}=8)$. Les différences sont analysées par le test de Student. Les significations $(\mathrm{p}<0,05)$ sont désignées par $*, a, b$ ou $c: *$ : éthanol vs témoin pour le même phénotype; $\mathrm{a}: \mathrm{Fa} /-v s$ fa/fa pour le même régime; $\mathrm{b}: \mathrm{Fa} /-v s$ Wistar pour le même régime ; $:$ fa/fa vs Wistar pour le même régime.

FIG. 1. - Composition en acides gras du foie.

FIG. 2. - Composition en acides gras du sang. L'acide gras $14: 0$ est présent dans tous les groupes de rats $(\%<1,2)$. Les acides gras $12: 0$ et $16: 1$ sont présents à l'état de traces.

FIG. 3. - Composition en acides gras du tissu adipeux. L'acide gras $14: 0$ est présent dans tous les groupes de rats $(\%: 1,1-1,5)$. Les acides gras $12: 0$ et $16: 1$ sont présents à l'état de traces. 
Avec le régime alcool, la composition en acides gras saturés et monoinsaturés du sang de rat obèse varie peu, par rapport au régime témoin. Cependant, la valeur du rapport $(16: 1)+(18: 1) /(16: 0)+(18: 0)$ diminue ; le taux d'acide linoléique augmente, celui de l'acide arachidonique diminue, comme la valeur du rapport (20:4) / (18:2).

Acides gras constitutifs du tissu adipeux (fig. 3). - A régime identique, le rat Zucker $\mathrm{fa} / \mathrm{fa}$ a un taux d'acide palmitique plus élevé que celui des rats témoins et un taux d'acide linoléique plus faible; avec les deux régimes, les valeurs des rapports $(16: 1)+(18: 1) /(16: 0)+(18: 0)$ sont plus faibles chez le rat obèse que chez les rats non obèses. Cependant, pour les 3 types de rats, le régime alcool ne provoque pas de variation de composition en acides gras du tissu adipeux, par rapport au régime témoin.

\section{Discussion.}

Au cours de l'ingestion chronique d'éthanol, la stéatose hépatique du rat normal n'est pas due à un phénomène de stress, comme on le rencontre lors d'une intoxication aiguë qui provoque une lipolyse adipocytaire (Lieber et al., 1966 ; Baraona et Lieber, 1979 ; Reitz, 1979). Une cause de cette stéatose est la diminution de l'oxydation hépatique des acides gras provoquant leur accumulation (Ontko, 1973 ; Lieber et Schmid, 1961 ; Lieber et al., 1967 ; Blomstrand et Kager, 1973). De plus, l'inhibition dans le foie du cycle des acides tricarboxyliques entraîne, lors de l'intoxication chronique par l'alcool, l'accumulation d'acétyl CoA, la formation d'acides gras et leur estérification sous forme de triacylglycérols (Nikkila et Ojala, 1963 ; Joly et al., 1973 ; Lamb et Fallon, 1974).

Acides gras du foie de rat Zucker fa/fa. - Avec un régime témoin, la lipogenèse est accrue dans le foie du rat obèse, par rapport à celle d'un rat témoin (Bray et York, 1979). II en résulte la formation de quantités élevées des acides palı̣̂itique et oléique (Kinkela et al., 1983) : la désaturation des acides gras est alors particulièrement active puisque la valeur du rapport $(16: 1)+(18: 1) /$ $(16: 0)+(18: 0)$ est plus élevée chez le rat fa/fa que chez le rat $\mathrm{Fa} /-(1,0$ et 0,4 respectivement). De telles variations sont retrouvées dans notre propre expérience.

Avec le régime alcool, la stéatose hépatique du rat obèse est moindre qu'avec le régime témoin (le poids du foie de rat $\mathrm{fa} / \mathrm{fa}$ est de $16,1 \mathrm{~g}$ avec le régime (E), de $20,5 \mathrm{~g}$ avec le régime $(T)$ (résultats non publiés)). Les deux principaux acides gras présents, l'acide palmitique et l'acide oléique, sont alors synthétisés en moindre quantité, du fait de la diminution de la lipogenèse hépatique. Celle-ci, évaluée par ailleurs, est en relation avec la diminution du taux de glucides du régime, en passant du régime témoin au régime éthanol. II en résulte que les pourcentages des autres principaux acides gras (acides stéarique, linoléique et arachidonique) augmentent. Par contre, les quantités totales de ces acides gras, calculées à partir des quantités de lipides hépatiques (résultats non publiés) sont toujours plus faibles avec le régime alcool qu'avec le régime témoin.

Ces variations sont inverses de celles qui sont décrites par Scheig et al. .1966) chez le rat Sprague-Dawley et par Thompson et Reitz (1978) chez le rat 
Long-Evans, lorsque ces rats sont soumis è une ingestion prolongée d'éthanol. Ce résultat paradoxal peut être expliqué par le comportement métabolique anormal du foie du rat obèse vis-à-vis des substrats glucidiques : l'accumulation de triacylglycérols dans le foie du rat fa/fa due à une lipogenèse intense est particulièrement importante avec un régime témoin (contenant $66 \%$ en poids d'amidon dans le régime) (résultats non publiés). Les quantités de triacylglycérols hépatiques sont alors respectivement de $1004 \mu$ moles chez le rat obèse et de 185 chez le rat témoin, les rats étant nourris par pair-feeding.

Acides gras du sang du rat Zucker fa/fa. - L'éthanol a un effet hypertriglycéridémiant chez le rat normal (Robinson et Quarfordt), 1981), mais il n'aggrave pas I'hyperlipémie du rat Zucker fa/fa (résultats non publiés). (L'hypertriglycéridémie des rats obèses au départ de l'expérience étant de 2,4 $\pm 0,2 \mathrm{mmoles} / \mathrm{I}$ atteint respectivement $10,1 \pm 1,0$ et $7,8 \pm 0,9$ mmoles/l après 4 semaines des régimes $\mathrm{T}$ et $\mathrm{E})$. De plus, le régime alcool entraîne, par rapport au régime témoin, l'augmentation de la valeur du rapport (20:4) / (18:2) dans le sang des rats témoins et la diminution de la valeur de ce rapport chez le rat Zucker obèse. Le rat obèse est spontanément hypertriglycéridémique. II produit une quantité exagérée de lipoprotéines de très faible densité (VLDL) véhiculant les acides gras en excès provenant du foie (Bray et York, 1979). Dans ces conditions, les acides gras du sang du rat obèse sont en grande partie d'origine hépatique et la composition en acides gras du sang et du foie de rat fa/fa sont donc comparables (le taux d'acide arachidonique est alors faible).

Acides gras du tissu adipeux du rat Zucker fa/fa. - La composition en acides gras constitutifs des lipides du tissu adipeux semble peu modifiée par l'apport d'alcool. Des différences entre les taux d'acides palmitique et linoléique des rats $\mathrm{fa} / \mathrm{fa}$ et $\mathrm{Fa} /$ - ou Wistar sont significatives avec le régime témoin ; elles le demeurent avec le régime alcool, sans que des variations se produisent entre les 2 régimes, pour un génotype donné.

L'origine des acides gras du tissu adipeux du rat obèse est variable selon l'environnement nutritionnel : avec un régime riche en glucides $168-69 \%$ de l'énergie) (Lemonnier et al., 1974 ; Kinkela et al., 1982) les acides gras du tissu adipeux du rat fa/fa proviennent essentiellement du foie, où la lipogenèse est accrue par rapport à celle du rat normal ; avec un régime pauvre en glucides (16 ou $39 \%$ de l'énergie respectivement) (selon les mêmes auteurs), la composition lipidique du tissu adipeux du rat fa/fa ressemble à celle du régime. Comme la valeur du taux de glucides de notre régime $(50 \%)$ est intermédiaire entre celles qui sont utilisées par les auteurs précédents (Lemonnier et al., 1974 ; Kinkela et al., 1983), il en résulte que les acides gras du tissu adipeux du rat obèse proviennent également du foie ou de l'alimentation.

Avec le régime alcool et par rapport au régime témoin, l'apport des acides gras au tissu adipeux serait diminué. En effet, le taux de lipides circulants est plus faible avec le régime alcool qu'avec le régime témoin. Cette constatation permettrait d'expliquer la plus faible évolution pondérale des animaux recevant le régime alcool, les variations de la masse corporelle étant dues principalement à celles du tissu adipeux. 


\section{Conclusion.}

Par rapport au régime témoin, le régime alcool entraîne une diminution des pourcentages des acides palmitique et oléique synthétisés par le foie du rat Zucker $\mathrm{fa} / \mathrm{fa}$, dont la stéatose est moins prononcée. L'hyperlipémie de ce rat n'est pas aggravée par l'éthanol et la composition en acides gras du sang reflète celle du foie. L'obésité du rat $\mathrm{fa} / \mathrm{fa}$, moins importante avec le régime alcool qu'avec le régime témoin, n'est pas caractérisée par des modifications qualitatives de composition en acides gras du tissu adipeux.

Reçu en juillet 1982.

Accepté en février 1983.

Remerciements. - Ce travail a été réalisé grâce à l'aide financière de I'INSERM (contrat de recherche libre $n^{\circ} 7852317$ ).

\section{Références}

BARAONA E., LIEBER C. S., 1979. Effects of ethanol on lipid metabolism. J. Lipid Res., 20, 289-315.

BEAUfAY H., AMAR-COSTESEC A., FEYTMANS E., THINES -SEMPOUX D., WIBO M., ROBI M., BERTHET J., 1974. Analytical study of microsomes and isolated subcellular membranes from rat liver. I. Biochemical methods. J. Cell Biol., 61, 188-212.

BLOMSTRAND R., KAGER L., 1973. The combustion of triolein $1-{ }^{14} \mathrm{C}$ and its inhibition by alcohol in man. Life Sci., 13, 113-123.

BRAY G. A., YORK D. A., 1979. Hypothalamic and genetic obesity in experimental animals : an autonomic and endocrine hypothesis. Physiol. Rev., 59, 719-809.

DE CARLI L. M., LIEBER C. S., 1967. Fatty liver in the rat after prolonged intake of ethanol with a nutritionally adequate new liquid diet. J. Nutr., 91, 331-336.

DELSAL J., 1944. Nouveau procédé d'extraction des lipides du sérum par le méthylal. Applications aux microdosages du cholestérol total, des phosphoaminolipides et des protéines. Bull. Soc. Chim. biol., 26, 99-105.

JOLY J. G., FEINMAN L., ISHII H., LIEBER C. S., 1973. Effect of chronic ethanol feeding on hepatic microsomal glycerophosphate acyltransferase activity. J. Lipid. Res., 14, 337-343.

KINKELA T., CHANUSSOT F., BACH A., MAX J. P., SCHIRARDIN H., DEBRY G., 1983. Effets de régimes à triacylglycérols à chaînes moyennes et à chaînes longues chez le rat génétiquement obèse Zucker $\mathrm{fa} / \mathrm{fa}$. Composition en acides gras et en triacylglycérols du foie et des tissus adipeux. Ann. Nutr. Metab., (sous presse).

LAMB R. G., FALLON H. J., 1974. An enzymatic explanation for dietary induced alterations in hepatic glycerolipid metabolism. Biochim. biophys. Acta, 348, 179-188.

LEMONNIER D., AUBERT R., SUQUET J. P., ROSSELIN G., 1974. Metabolism of genetically obese rats on normal or high fat diet. Diabetologia, 10, 697-701.

LIEBER C. S., JONES D. P., DE CARLI L. M., 1965. Effects of prolonged ethanol intake : production of fatty liver despite adequate diets. J. clin. Invest., 44, 1009-1021.

LIEBER C. S., LEFEVRE A., SPRITZ N., FEINMAN L., DE CARLI L. M., 1967 : Difference in hepatic metabolism of long and medium-chain fatty acids : the role of fatty acid chain length in the production of alcoholic fatty liver. J. clin. Invest., 46, 1451-1460.

LIEBER C. S., SCHMID R., 1961. The effect of ethanol on fatty acid synthesis in vitro. J. clin. Invest., 40, 394-399. 
LIEBER C. S., SPRITZ N., DE CARLI L. M., 1966. Role of dietary, adipose and endogenously synthetized fatty acids in the pathogenesis of the alcoholic fatty liver. J. clin. Invest., 45, 1400-1411.

MORRISON W. R., SMITH L. M., 1964. Preparation of fatty acid methyl esters and dimethylacetals from lipids with boron fluoride-methanol. I. Lipid Res., 5, 600-608.

NIKKILA E. A., OJALA K., 1963. Role of hepatic L-glycerophosphate and triglyceride synthesis in the production of fatty liver by ethanol. Proc. Soc. exp. Biol. Med., 113, 814-817.

ONTKO J. A., 1973. Effects of ethanol on the metabolism of free fatty acids in isolated liver cells. J. Lipid Res., 14, 78-85.

REITZ R. C., 1979. The effects of ethanol ingestion on lipid metabolism. Progr. Lipid Res., 18, 87-115.

ROBINSON S. F., QUARFORDT S. H., 1981. The effect of ethanol on lipoprotein metabolism. Alcoholism, 5, 101-109.

SCHEIG R., ALEXANDER N. M., KLATSKIN G., 1966. Effects of prolonged ingestion of glucose or ethanol on tissue lipid composition and lipid biosynthesis in rat. J. Lipid Res., 7, 188-196.

THOMPSON J. A., REITZ R. C., 1978. Effects of ethanol ingestion and dietary fat levels on mitochrondrial lipids in male and female rats. Lipids, 13, 540-550. 